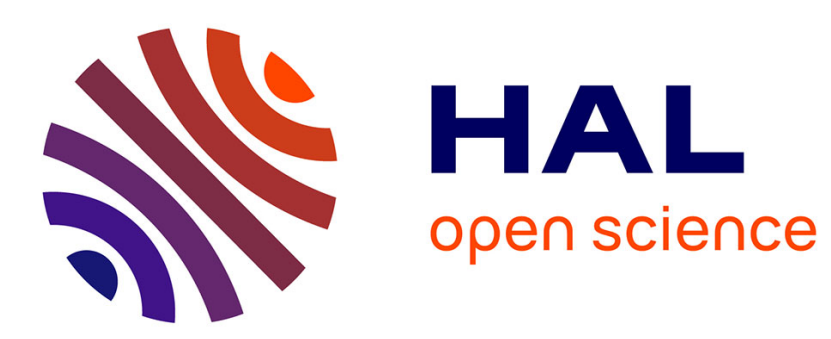

\title{
Effects of permanent dipole moments in degenerate four-wave-mixing processes
}

\author{
Jean-Pascal Lavoine, Claudine Hoerner, Albert A. Villaeys
}

\section{To cite this version:}

Jean-Pascal Lavoine, Claudine Hoerner, Albert A. Villaeys. Effects of permanent dipole moments in degenerate four-wave-mixing processes. Physical Review A: Atomic, molecular, and optical physics [1990-2015], 1991, 44 (9), pp.5947-5957. 10.1103/PhysRevA.44.5947 . hal-00880756

\section{HAL Id: hal-00880756 https://hal.science/hal-00880756}

Submitted on 7 Nov 2013

HAL is a multi-disciplinary open access archive for the deposit and dissemination of scientific research documents, whether they are published or not. The documents may come from teaching and research institutions in France or abroad, or from public or private research centers.
L'archive ouverte pluridisciplinaire HAL, est destinée au dépôt et à la diffusion de documents scientifiques de niveau recherche, publiés ou non, émanant des établissements d'enseignement et de recherche français ou étrangers, des laboratoires publics ou privés. 


\title{
Effects of permanent dipole moments in degenerate four-wave-mixing processes
}

\author{
J. P. Lavoine, C. Hoerner, and A. A. Villaeys \\ Groupe d'Optique Nonlinéaire et d'Optoélectronique, Institut de Physique et Chimie des Matériaux \\ de Strasbourg, 5, rue de l'Université, 67084 Strasbourg CEDEX, France
}

(Received 2 April 1991)

\begin{abstract}
The main goal of this paper is to analyze a two-photon degenerate four-wave-mixing process that occurs in an isotropic media modeled by homogeneously broadened two-level systems having unequal permanent dipole moments and subject to rotational diffusion. Our theoretical treatment is based upon a third-order perturbative solution of the density-matrix equations and does not require the rotating-wave approximation. The medium being excited by three stationary fields, we give an analytical expression of the macroscopic polarization up to the third order, including permanent dipole moments, orientational diffusion, and field polarizations. We apply these general results to the description of a degenerate fourwave-mixing process by selecting, in the expressions, the contributions in the direction $\mathbf{k}_{\alpha}-\mathbf{k}_{\beta}+\mathbf{k}_{\gamma}$, $\mathbf{k}_{\alpha}, \mathbf{k}_{\beta}$, and $\mathbf{k}_{\gamma}$ being, respectively, the wave vectors of the exciting fields. We show that the two-photon degenerate four-wave mixing is dependent on the permanent dipole moments and is not present if these dipole moments are equal or zero. We analyze the influence of rotational diffusion. Different cases of field polarizations are also considered, and we discuss a comparison of the well-known one-photon degenerate four-wave-mixing process and this two-photon process. This process is also discussed in the particular case of phase conjugation.
\end{abstract}

PACS number(s): 42.65.Ma, 42.65.Hw

\section{INTRODUCTION}

Molecules whose states have permanent dipole moments have been the subject of many works in recent years. These molecules can exhibit nonlinear-optical properties that differ substantially from molecules having parity symmetry. In particular, two-photon absorption can occur in two-level systems having unequal permanent dipoles $[1,2]$. The effect of permanent dipole moments on the transient and steady-state transition probabilities of a two-level system interacting with either a monochromatic field, two optical fields of different frequencies, or an optical field in the presence of a static electric field, has been the subject of many papers [3-5]. Recently Bavli and co-workers $[6,7]$ have calculated the transition rates, and the linear- and nonlinear-optical properties of a homogeneously broadened two-level system with permanent dipole moments in the presence of two pump fields. They have showed that even in the case of a two-level system with permanent dipole moments, Raman scattering, two-photon absorption, and wave mixing of arbitrary order can be observed when the permanent dipole moments of the two levels are not equal.

The purpose of this work is to analyze a degenerate four-wave-mixing process in a medium modeled by twolevel systems having unequal permanent dipole moments. As already mentioned, two-photon absorption can occur in such systems. Therefore it is reasonable to think that two-photon degenerate four-wave mixing can also appear in these media. Optical phase conjugation by two-photon degenerate four-wave mixing has been the subject of different works $[8,9]$, but usually the medium is described by a three-level system that has nonpermanent dipole moments. In our model we consider that three optical fields of the same frequency interact with homogeneously broadened two-level systems having unequal permanent dipole moments. We assume that the molecules in the medium are subject to rotational diffusion. The medium polarization is calculated by using the density-matrix equations, which are solved to third order by perturbational techniques. In these equations the orientational effects are taken into account by a phenomenological diffusion term. We shall assume a uniform distribution of the dipole moment in the medium. This means that the probability of finding the dipole moment of the molecules in a given direction will be constant. This situation is generally encountered with noninteracting molecules in a nonpolar solvent and when no static field is present. Our treatment does not make the rotating-wave approximation. This is of particular importance here because a nonlinear effect is expected far from the resonance frequency. Our theory enables us to evaluate the macroscopic third-order polarization of the medium. We show that a two-photon degenerate four-wave mixing occurs when the field frequencies are equal to half of the resonance frequency. We discuss the influence of permanent dipole moments on this process which does not exist when the permanent dipole moments are equal or zero. We analyze the effect of rotational diffusion and the role of the field polarizations on this nonlinear optical process.

\section{THEORY}

The nonlinear medium of interest here is modeled by an ensemble of homogeneously broadened two-level systems. Each of them is characterized by a dipole moment $\boldsymbol{\mu}$, and longitudinal and transverse decay rates $\Gamma_{1}$ and $\Gamma_{2}$, 
respectively. Finally, the transition energy is denoted by $\hbar \omega_{21}$. We assume that the levels of the microscopic twolevel systems have permanent dipole moments so that $\boldsymbol{\mu}$ can be written as

$$
\boldsymbol{\mu}=\left[\begin{array}{ll}
\boldsymbol{\mu}_{11} & \boldsymbol{\mu}_{12} \\
\boldsymbol{\mu}_{21} & \boldsymbol{\mu}_{22}
\end{array}\right)
$$

with $\boldsymbol{\mu}_{11} \neq \boldsymbol{\mu}_{22}$. To simplify our model, we consider that the permanent dipole moment and the transition dipole moment are parallel. This situation can be encountered in different molecules [10]. Therefore, we can define a unit vector $\epsilon_{\mu}(\theta, \varphi)$ in the direction of $\boldsymbol{\mu}$, where $\theta$ and $\varphi$ are the orientational coordinates of $\mu$ defined with respect to the laboratory frame. We have the relation

$$
\boldsymbol{\mu}_{i j}=\boldsymbol{\epsilon}_{\mu} \mu_{i j}
$$

For the sake of simplicity, we omit, when possible, the explicit dependence in the mathematical expressions. For example, in relation (2), $\mu$ and $\epsilon_{\mu}$ must be read as $\mu(\theta, \varphi)$ and $\epsilon_{\mu}(\theta, \varphi)$, respectively.

The material system described above interacts with three electric fields. Let us call $\mathbf{E}(\mathbf{r}, t)$ the total field. It can be expressed as

$$
\mathbf{E}(\mathbf{r}, t)=\sum_{\alpha} \mathbf{E}_{\alpha}(\mathbf{r}, t), \quad \alpha=1,2,3,
$$

where $\mathbf{E}_{\alpha}(\mathbf{r}, t)$ are the individual fields that are taken in the form

$$
\mathbf{E}_{\alpha}(\mathbf{r}, t)=\frac{1}{2} \epsilon_{\alpha} \mathscr{E}_{\alpha} \exp \left[i\left(\omega_{\alpha} t-\mathbf{k}_{\alpha} \cdot \mathbf{r}\right)\right]+\text { c.c. . }
$$

Here c.c. stands for the complex conjugate part and $\epsilon_{\alpha}$ is a unit polarization vector. For the sake of convenience, we will assume all along that the perturbational approach is valid and use the third-order iterative solution for the density matrix. This solution includes a possible orientational diffusion mechanism that will be relevant for our purpose. It is also assumed that the orientational diffusion results from statistical processes that are independent of the applied fields. This process can be handled by including a rotational diffusion term in the equation of motion of the density-matrix elements [11]. To simplify, the diffusion constants $D$ are chosen to be identical for the various states of the system and the transverse decay rate $\Gamma_{2}$ is assumed to be very large compared to $D$ [12]. With this assumption in mind, the evolution of the density-matrix elements for a given value of $\theta$ and $\varphi$ is given by the relations [12]

$$
\begin{aligned}
& \frac{\partial \rho_{11}}{\partial t}(\mathbf{r}, t, \theta, \varphi)= \frac{-i}{\hbar}\left\{\rho_{12}(\mathbf{r}, t, \theta, \varphi)\left[\boldsymbol{\mu}_{21}(\theta, \varphi) \cdot \mathbf{E}(\mathbf{r}, t)\right]-\left[\boldsymbol{\mu}_{12}(\theta, \varphi) \cdot \mathbf{E}(\mathbf{r}, t)\right] \rho_{21}(\mathbf{r}, t, \theta, \varphi)\right\} \\
&+D \Delta_{\theta, \varphi} \rho_{11}(\mathbf{r}, t, \theta, \varphi)+\Gamma_{1} \rho_{22}(\mathbf{r}, t, \theta, \varphi) \\
& \frac{\partial \rho_{22}}{\partial t}(\mathbf{r}, t, \theta, \varphi)= \frac{i}{\hbar}\left\{\rho_{12}(\mathbf{r}, t, \theta, \varphi)\left[\boldsymbol{\mu}_{21}(\theta, \varphi) \cdot \mathbf{E}(\mathbf{r}, t)\right]-\left[\boldsymbol{\mu}_{12}(\theta, \varphi) \cdot \mathbf{E}(\mathbf{r}, t)\right] \rho_{21}(\mathbf{r}, t, \theta, \varphi)\right\} \\
&+D \Delta_{\theta, \varphi} \rho_{22}(\mathbf{r}, t, \theta, \varphi)-\Gamma_{1} \rho_{22}(\mathbf{r}, t, \theta, \varphi) \\
& \frac{\partial \rho_{12}}{\partial t}(\mathbf{r}, t, \theta, \varphi)=-\left(\Gamma_{2}-i \omega_{21}\right) \rho_{12}(\mathbf{r}, t, \theta, \varphi)-\frac{i}{\hbar}[\mathbf{d} \cdot \mathbf{E}(\mathbf{r}, t)] \rho_{12}(\mathbf{r}, t, \theta, \varphi) \\
&+\frac{i}{\hbar}\left[\mu_{12} \cdot \mathbf{E}(\mathbf{r}, t)\right]\left[\rho_{22}(\mathbf{r}, t, \theta, \varphi)-\rho_{11}(\mathbf{r}, t, \theta, \varphi)\right] \\
& \rho_{21}(\mathbf{r}, t, \theta, \varphi)=\rho_{12}^{*}(\mathbf{r}, t, \theta, \varphi),
\end{aligned}
$$

where $\rho_{11}(\mathbf{r}, t, \theta, \varphi)$ and $\rho_{22}(\mathbf{r}, t, \theta, \varphi)$ are the diagonal elements of the density matrix $\rho$. The nondiagonal elements are given by $\rho_{12}(\mathbf{r}, t, \theta, \varphi)$ and $\rho_{21}(\mathbf{r}, t, \theta, \varphi)$ and correspond to the coherence between the excited state 2 and the ground state 1. $\Delta_{\theta, \varphi}$ is the Laplace operator in the spherical coordinates. The quantity $\mathbf{d}$ is the difference between the permanent dipole moments of the ground and excited states, that is to say,

$$
\mathbf{d}(\theta, \varphi)=\boldsymbol{\mu}_{22}(\theta, \varphi)-\boldsymbol{\mu}_{11}(\theta, \varphi) .
$$

It is important to note that the dephasing constant $\Gamma_{2}^{-1}$ includes pure dephasing effects and that our description does not introduce the rotating-wave approximation.

As already mentioned, the set of equations (5)-(8) will be solved by using a perturbative treatment. Therefore, at this step of the calculation, we can first make a remark on the influence of the permanent dipole moment. As no- ticed by different authors [13], when $\mathbf{d}$ is zero, the iterative solutions to optical Bloch equations for two-level models reveal a very peculiar structure that is not valid anymore for many-level systems. The contributions to the populations and coherences are decoupled and depend on the order of the perturbation. For instance, the even orders give corrections to the populations only, while the odd orders contribute to the coherence only. Equations (5)-(8) show clearly that this peculiar structure will vanish when $\mathbf{d}$ will be nonzero.

Let us solve now Eqs. (5)-(8) up to the third order. In the zeroth-order approximation, we have

$$
\frac{\partial \rho_{11}^{(0)}}{\partial t}(\mathbf{r}, t, \theta, \varphi)=\Gamma_{1} \rho_{22}^{(0)}(\mathbf{r}, t, \theta, \varphi)+D \Delta_{\theta, \varphi} \rho_{11}^{(0)}(\mathbf{r}, t, \theta, \varphi)
$$


$\frac{\partial \rho_{22}^{(0)}}{\partial t}(\mathbf{r}, t, \theta, \varphi)=-\Gamma_{1} \rho_{22}^{(0)}(\mathbf{r}, t, \theta, \varphi)+D \Delta_{\theta, \varphi} \rho_{22}^{(0)}(\mathbf{r}, t, \theta, \varphi)$

$\frac{\partial \rho_{12}^{(0)}}{\partial t}(\mathbf{r}, t, \theta, \varphi)=-\left(\Gamma_{2}-i \omega_{21}\right) \rho_{12}^{(0)}(\mathbf{r}, t, \theta, \varphi)$

Solutions to these equations are straightforwardly obtained if the initial conditions are well defined. For the purpose of this work, we will assume that, at $t=-\infty$, the system is in equilibrium. This means $\rho_{11}(-\infty)=1$, $\rho_{22}(-\infty)=0$, and $\rho_{12}(-\infty)=0$. Then by expanding $\rho_{11}^{(0)}(\mathbf{r}, t, \theta, \varphi)$ and $\rho_{22}^{(0)}(\mathbf{r}, t, \theta, \varphi)$ over a spherical harmonics basis, the zeroth-order approximation gives the result

$$
\begin{aligned}
& \rho_{12}^{(0)}(\mathbf{r}, t, \theta, \varphi)=\rho_{22}^{(0)}(\mathbf{r}, t, \theta, \varphi)=0, \\
& \rho_{11}^{(0)}(\mathbf{r}, t, \theta, \varphi)=1 .
\end{aligned}
$$

Next we consider the first-order approximation. The dynamical equations take the form

$$
\frac{\partial \rho_{11}^{(1)}}{\partial t}(\mathbf{r}, t, \theta, \varphi)=\Gamma_{1} \rho_{22}^{(1)}(\mathbf{r}, t, \theta, \varphi)+D \Delta_{\theta, \varphi} \rho_{11}^{(1)}(\mathbf{r}, t, \theta, \varphi),
$$

$$
\frac{\partial \rho_{22}^{(1)}}{\partial t}(\mathbf{r}, t, \theta, \varphi)=-\Gamma_{1} \rho_{22}^{(1)}(\mathbf{r}, t, \theta, \varphi)+D \Delta_{\theta, \varphi} \rho_{22}^{(1)}(\mathbf{r}, t, \theta, \varphi)
$$

$$
\begin{aligned}
\frac{\partial \rho_{12}^{(1)}}{\partial t}(\mathbf{r}, t, \theta, \varphi)= & -\left(\Gamma_{2}-i \omega_{21}\right) \rho_{12}^{(1)}(\mathbf{r}, t, \theta, \varphi) \\
& -\frac{i}{\hbar}\left[\boldsymbol{\mu}_{12}(\theta, \varphi) \cdot \mathbf{E}(\mathbf{r}, t)\right]
\end{aligned}
$$

At the initial time there is no first-order correction. This implies that for Eqs. (15) and (16) we have

$$
\rho_{11}^{(1)}(\mathbf{r}, t, \theta, \varphi)=\rho_{22}^{(1)}(\mathbf{r}, t, \theta, \varphi)=0 .
$$

The solution of Eq. (17) is

$$
\begin{aligned}
\rho_{12}^{(1)}(\mathbf{r}, t, \theta, \varphi)=\frac{-i}{\hbar} \int_{-\infty}^{t} & {\left[\boldsymbol{\mu}_{12}(\theta, \varphi) \cdot \mathbf{E}\left(\mathbf{r}, t_{1}\right)\right] } \\
& \times \exp \left[-\left(\Gamma_{2}-i \omega_{21}\right)\left(t-t_{1}\right)\right] d t_{1} .
\end{aligned}
$$

Introducing the expression of $\mathbf{E}(\mathbf{r}, t)$ given by relations (3) and (4), the coherence to the first order takes the form

$$
\rho_{12}^{(1)}(\mathbf{r}, t, \theta, \varphi)=-\frac{i}{2 \hbar} \mu_{12} \sum_{\alpha}\left[\frac{\left[\boldsymbol{\epsilon}_{\mu}(\theta, \varphi) \cdot \boldsymbol{\epsilon}_{\alpha}\right]}{\Gamma_{2}+i\left(\omega_{\alpha}-\omega_{21}\right)} \mathscr{E}_{\alpha} \exp \left[i\left(\omega_{\alpha} t-\mathbf{k}_{\alpha} \cdot \mathbf{r}\right)\right]+\frac{\left[\boldsymbol{\epsilon}_{\mu}(\theta, \varphi) \cdot \boldsymbol{\epsilon}_{\alpha}^{*}\right]}{\Gamma_{2}-i\left(\omega_{\alpha}+\omega_{21}\right)} \mathscr{E}_{\alpha}^{*} \exp \left[-i\left(\omega_{\alpha} t-\mathbf{k}_{\alpha} \cdot \mathbf{r}\right)\right]\right]
$$

We consider now the second-order contributions to populations and coherences. The starting equations are

$$
\begin{aligned}
\frac{\partial \rho_{11}^{(2)}}{\partial t}(\mathbf{r}, t, \theta, \varphi)= & \frac{-i}{\hbar}\left\{\rho_{12}^{(1)}(\mathbf{r}, t, \theta, \varphi)\left[\boldsymbol{\mu}_{21}(\theta, \varphi) \cdot \mathbf{E}(\mathbf{r}, t)\right]-\left[\boldsymbol{\mu}_{12}(\theta, \varphi) \cdot \mathbf{E}(\mathbf{r}, t)\right] \rho_{21}^{(1)}(\mathbf{r}, t, \theta, \varphi)\right\} \\
& +D \Delta_{\theta, \varphi} \rho_{11}^{(2)}(\mathbf{r}, t, \theta, \varphi)+\Gamma_{1} \rho_{22}^{(2)}(\mathbf{r}, t, \theta, \varphi) \\
\frac{\partial \rho_{22}^{(2)}}{\partial t}(\mathbf{r}, t, \theta, \varphi)= & \frac{i}{\hbar}\left\{\rho_{12}^{(1)}(\mathbf{r}, t, \theta, \varphi)\left[\mu_{21}(\theta, \varphi) \cdot \mathbf{E}(\mathbf{r}, t)\right]-\left[\boldsymbol{\mu}_{12}(\theta, \varphi) \cdot \mathbf{E}(\mathbf{r}, t)\right] \rho_{21}^{(1)}(\mathbf{r}, t, \theta, \varphi)\right\} \\
& +D \Delta_{\theta, \varphi} \rho_{22}^{(2)}(\mathbf{r}, t, \theta, \varphi)-\Gamma_{1} \rho_{22}^{(2)}(\mathbf{r}, t, \theta, \varphi),
\end{aligned}
$$

$\frac{\partial \rho_{12}^{(2)}}{\partial t}(\mathbf{r}, t, \theta, \varphi)=-\left(\Gamma_{2}-i \omega_{21}\right) \rho_{12}^{(2)}(\mathbf{r}, t, \theta, \varphi)-\frac{i}{\hbar}[\mathbf{d} \cdot \mathbf{E}(\mathbf{r}, t)] \rho_{12}^{(1)}(\mathbf{r}, t, \theta, \varphi)+\frac{i}{\hbar}\left[\boldsymbol{\mu}_{12} \cdot \mathbf{E}(\mathbf{r}, t)\right]\left[\rho_{22}^{(1)}(\mathbf{r}, t, \theta, \varphi)-\rho_{11}^{(1)}(\mathbf{r}, t, \theta, \varphi)\right]$

Introducing the first-order corrections previously determined, Eq. (23) is easily solved and the contribution to the second-order coherences is given by

$$
\begin{aligned}
\rho_{12}^{(2)}(\mathbf{r}, t, \theta, \varphi)=-\frac{d \mu_{12}}{4 \hbar^{2}} \sum_{\alpha, \beta} & \left\{a_{\alpha \beta}\left[\boldsymbol{\epsilon}_{\mu}(\theta, \varphi) \cdot \boldsymbol{\epsilon}_{\alpha}\right]\left[\boldsymbol{\epsilon}_{\mu}(\theta, \varphi) \cdot \boldsymbol{\epsilon}_{\beta}\right] \mathscr{E}_{\alpha} \mathscr{E}_{\beta} \exp \left[i\left(\omega_{\alpha}+\omega_{\beta}\right) t\right] \exp \left[-i\left(\mathbf{k}_{\alpha}+\mathbf{k}_{\beta}\right) \cdot \mathbf{r}\right]\right. \\
& +b_{\alpha \beta}\left[\boldsymbol{\epsilon}_{\mu}(\theta, \varphi) \cdot \boldsymbol{\epsilon}_{\alpha}\right]\left[\boldsymbol{\epsilon}_{\mu}(\theta, \varphi) \cdot \boldsymbol{\epsilon}_{\beta}^{*}\right] \mathscr{E}_{\alpha} \mathscr{E}_{\beta}^{*} \exp \left[i\left(\omega_{\alpha}-\omega_{\beta}\right) t\right] \exp \left[-i\left(\mathbf{k}_{\alpha}-\mathbf{k}_{\beta}\right) \cdot \mathbf{r}\right] \\
& \left.+c_{\alpha \beta}\left[\boldsymbol{\epsilon}_{\mu}(\theta, \varphi) \cdot \boldsymbol{\epsilon}_{\alpha}^{*}\right]\left[\boldsymbol{\epsilon}_{\mu}(\theta, \varphi) \cdot \boldsymbol{\epsilon}_{\beta}^{*}\right] \mathscr{E}_{\alpha}^{*} \mathscr{E}_{\beta}^{*} \exp \left[-i\left(\omega_{\alpha}+\omega_{\beta}\right) t\right] \exp \left[i\left(\mathbf{k}_{\alpha}+\mathbf{k}_{\beta}\right) \cdot \mathbf{r}\right]\right\}
\end{aligned}
$$

where $a_{\alpha \beta}, b_{\alpha \beta}$, and $c_{\alpha \beta}$ are defined by the relations

$$
a_{\alpha \beta}=\frac{1}{\left[\Gamma_{2}+i\left(\omega_{\alpha}-\omega_{21}\right)\right]\left[\Gamma_{2}+i\left(\omega_{\alpha}+\omega_{\beta}-\omega_{21}\right)\right]},
$$




$$
\begin{aligned}
& b_{\alpha \beta}=\left(\frac{1}{\left[\Gamma_{2}+i\left(\omega_{\alpha}-\omega_{21}\right)\right]}+\frac{1}{\left[\Gamma_{2}-i\left(\omega_{\beta}+\omega_{21}\right)\right]}\right] \frac{1}{\left[\Gamma_{2}+i\left(\omega_{\alpha}-\omega_{\beta}-\omega_{21}\right)\right]}, \\
& c_{\alpha \beta}=\frac{1}{\left[\Gamma_{2}-i\left(\omega_{\alpha}+\omega_{21}\right)\right]\left[\Gamma_{2}-i\left(\omega_{\alpha}+\omega_{\beta}+\omega_{21}\right)\right]} .
\end{aligned}
$$

Expanding $\rho_{22}^{(2)}(\mathbf{r}, t, \theta, \varphi)$ over a spherical harmonics basis, and introducing this development into Eq. (22), $\rho_{22}^{(2)}(\mathbf{r}, t, \theta, \varphi)$ can be expressed as

$$
\begin{array}{r}
\rho_{22}^{(2)}(\mathbf{r}, t, \theta, \varphi)=\frac{i}{\hbar} \sum_{l, m} \int_{-\infty}^{t} d t_{1} \int_{0}^{2 \pi} d \varphi_{1} \int_{0}^{\pi} d \theta_{1} \sin \theta_{1}\left\{\rho_{12}^{(1)}\left(\mathbf{r}, t_{1}, \theta_{1}, \varphi_{1}\right)\left[\boldsymbol{\mu}_{21}\left(\theta_{1}, \varphi_{1}\right) \cdot \mathbf{E}\left(\mathbf{r}, t_{1}\right)\right]\right. \\
\left.-\left[\boldsymbol{\mu}_{12}\left(\theta_{1}, \varphi_{1}\right) \cdot \mathbf{E}\left(\mathbf{r}, t_{1}\right)\right] \rho_{21}^{(1)}\left(\mathbf{r}, \mathrm{t}_{1}, \theta_{1}, \varphi_{1}\right)\right\} \\
\times Y_{l m}^{*}\left(\theta_{1}, \varphi_{1}\right) Y_{l m}(\theta, \varphi) \exp \left\{-\left[\Gamma_{1}+D l(l+1)\right]\left(t-t_{1}\right)\right\},
\end{array}
$$

where $Y_{l m}(\theta, \varphi)$ are the spherical harmonics. By carrying out the integrations in the previous relation, $\rho_{22}^{(2)}(\mathbf{r}, t, \theta, \varphi)$ takes the form

$$
\begin{aligned}
\rho_{22}^{(2)}(\mathbf{r}, t, \theta, \varphi)=\frac{\left|\mu_{12}\right|^{2}}{4 \hbar^{2}} \sum_{\alpha, \beta} & \left(\left\{d_{\alpha \beta}^{(1)}\left[\boldsymbol{\epsilon}_{\mu}(\theta, \varphi) \cdot \boldsymbol{\epsilon}_{\alpha}\right]\left[\boldsymbol{\epsilon}_{\mu}(\theta, \varphi) \cdot \boldsymbol{\epsilon}_{\beta}\right]+\frac{1}{3} d_{\alpha \beta}^{(2)}\left(\boldsymbol{\epsilon}_{\alpha} \cdot \boldsymbol{\epsilon}_{\beta}\right)\right\} \mathscr{E}_{\alpha} \mathscr{E}_{\beta} \exp \left[i\left(\omega_{\alpha}+\omega_{\beta}\right) t\right] \exp \left[-i\left(\mathbf{k}_{\alpha}+\mathbf{k}_{\beta}\right) \cdot \mathbf{r}\right]\right. \\
+ & \left\{e_{\alpha \beta}^{(1)}\left[\boldsymbol{\epsilon}_{\mu}(\theta, \varphi) \cdot \boldsymbol{\epsilon}_{\alpha}\right]\left[\boldsymbol{\epsilon}_{\mu}(\theta, \varphi) \cdot \boldsymbol{\epsilon}_{\beta}^{*}\right]+\frac{1}{3} e_{\alpha \beta}^{(2)}\left(\boldsymbol{\epsilon}_{\alpha} \cdot \boldsymbol{\epsilon}_{\beta}^{*}\right)\right\} \mathscr{E}_{\alpha} \mathscr{E}_{\beta}^{*} \exp \left[i\left(\omega_{\alpha}-\omega_{\beta}\right) t\right] \exp \left[-i\left(\mathbf{k}_{\alpha}-\mathbf{k}_{\beta}\right) \cdot \mathbf{r}\right] \\
+ & \left.\left\{f_{\alpha \beta}^{(1)}\left[\boldsymbol{\epsilon}_{\mu}(\theta, \varphi) \cdot \epsilon_{\alpha}^{*}\right]\left[\boldsymbol{\epsilon}_{\mu}(\theta, \varphi) \cdot \epsilon_{\beta}^{*}\right]+\frac{1}{3} f_{\alpha \beta}^{(2)}\left(\boldsymbol{\epsilon}_{\alpha}^{*} \cdot \epsilon_{\beta}^{*}\right)\right\} \mathscr{E}_{\alpha}^{*} \mathscr{E}_{\beta}^{*} \exp \left[-i\left(\omega_{\alpha}+\omega_{\beta}\right) t\right] \exp \left[i\left(\mathbf{k}_{\alpha}+\mathbf{k}_{\beta}\right) \cdot \mathbf{r}\right]\right)
\end{aligned}
$$

where we have defined the quantities

$$
\begin{aligned}
d_{\alpha \beta}^{(1)}= & {\left[\frac{1}{\Gamma_{2}+i\left(\omega_{\alpha}-\omega_{21}\right)}+\frac{1}{\Gamma_{2}+i\left(\omega_{\alpha}+\omega_{21}\right)}\right] \frac{1}{\Gamma_{1}+6 D+i\left(\omega_{\alpha}+\omega_{\beta}\right)}, } \\
d_{\alpha \beta}^{(2)}= & {\left[\frac{1}{\Gamma_{2}+i\left(\omega_{\alpha}-\omega_{21}\right)}+\frac{1}{\Gamma_{2}+i\left(\omega_{\alpha}+\omega_{21}\right)}\right]\left[\frac{1}{\Gamma_{1}+i\left(\omega_{\alpha}+\omega_{\beta}\right)}-\frac{1}{\Gamma_{1}+6 D+i\left(\omega_{\alpha}+\omega_{\beta}\right)}\right], } \\
e_{\alpha \beta}^{(1)}= & {\left[\frac{1}{\Gamma_{2}+i\left(\omega_{\alpha}-\omega_{21}\right)}+\frac{1}{\Gamma_{2}-i\left(\omega_{\beta}+\omega_{21}\right)}+\frac{1}{\Gamma_{2}-i\left(\omega_{\beta}-\omega_{21}\right)}+\frac{1}{\Gamma_{2}+i\left(\omega_{\alpha}+\omega_{21}\right)}\right] \frac{1}{\Gamma_{1}+6 D+i\left(\omega_{\alpha}-\omega_{\beta}\right)}, } \\
e_{\alpha \beta}^{(2)}= & {\left[\frac{1}{\Gamma_{2}+i\left(\omega_{\alpha}-\omega_{21}\right)}+\frac{1}{\Gamma_{2}-i\left(\omega_{\beta}+\omega_{21}\right)}+\frac{1}{\Gamma_{2}-i\left(\omega_{\beta}-\omega_{21}\right)}+\frac{1}{\Gamma_{2}+i\left(\omega_{\alpha}+\omega_{21}\right)}\right] } \\
& \times\left[\frac{1}{\Gamma_{1}+i\left(\omega_{\alpha}-\omega_{\beta}\right)}-\frac{1}{\Gamma_{1}+6 D+i\left(\omega_{\alpha}-\omega_{\beta}\right)}\right], \\
f_{\alpha \beta}^{(1)}= & {\left[\frac{1}{\Gamma_{2}-i\left(\omega_{\alpha}+\omega_{21}\right)}+\frac{1}{\Gamma_{2}-i\left(\omega_{\alpha}-\omega_{21}\right)}\right] \frac{1}{\Gamma_{1}+6 D-i\left(\omega_{\alpha}+\omega_{\beta}\right)}, } \\
f_{\alpha \beta}^{(2)}= & {\left[\frac{1}{\Gamma_{2}-i\left(\omega_{\alpha}+\omega_{21}\right)}+\frac{1}{\Gamma_{2}-i\left(\omega_{\alpha}-\omega_{21}\right)}\right]\left[\frac{1}{\Gamma_{1}-i\left(\omega_{\alpha}+\omega_{\beta}\right)}-\frac{1}{\Gamma_{1}+6 D-i\left(\omega_{\alpha}+\omega_{\beta}\right)}\right] }
\end{aligned}
$$

Finally, the last relation is given by

$$
\rho_{11}^{(2)}(\mathbf{r}, t, \theta, \varphi)=-\rho_{22}^{(2)}(\mathbf{r}, t, \theta, \varphi)
$$

It must be noted that the coefficients $d_{\alpha \beta}^{(2)}, e_{\alpha \beta}^{(2)}, f_{\alpha \beta}^{(2)}$, are equal to zero if no rotational diffusion takes place in the medium.

We are now ready to evaluate the third-order contribution, which is the main goal of the present calculation. The 
starting equations are

$$
\begin{aligned}
\frac{\partial \rho_{11}^{(3)}}{\partial t}(\mathbf{r}, t, \theta, \varphi)= & \frac{-i}{\hbar}\left\{\rho_{12}^{(2)}(\mathbf{r}, t, \theta, \varphi)\left[\boldsymbol{\mu}_{21}(\theta, \varphi) \cdot \mathbf{E}(\mathbf{r}, t)\right]-\left[\boldsymbol{\mu}_{12}(\theta, \varphi) \cdot \mathbf{E}(\mathbf{r}, t)\right] \rho_{21}^{(2)}(\mathbf{r}, t, \theta, \varphi)\right\} \\
& +D \Delta_{\theta, \varphi} \rho_{11}^{(3)}(\mathbf{r}, t, \theta, \varphi)+\Gamma_{1} \rho_{22}^{(3)}(\mathbf{r}, t, \theta, \varphi) \\
\frac{\partial \rho_{22}^{(3)}}{\partial t}(\mathbf{r}, t, \theta, \varphi)= & \frac{i}{\hbar}\left\{\rho_{12}^{(2)}(\mathbf{r}, t, \theta, \varphi)\left[\mu_{21}(\theta, \varphi) \cdot \mathbf{E}(\mathbf{r}, t)\right]-\left[\mu_{12}(\theta, \varphi) \cdot \mathbf{E}(\mathbf{r}, t)\right] \rho_{21}^{(2)}(\mathbf{r}, t, \theta, \varphi)\right\} \\
& +D \Delta_{\theta, \varphi} \rho_{22}^{(3)}(\mathbf{r}, t, \theta, \varphi)-\Gamma_{1} \rho_{22}^{(3)}(\mathbf{r}, t, \theta, \varphi) \\
\frac{\partial \rho_{12}^{(3)}}{\partial t}(\mathbf{r}, t, \theta, \varphi)= & -\left(\Gamma_{2}-i \omega_{21}\right) \rho_{12}^{(3)}(\mathbf{r}, t, \theta, \varphi)-\frac{i}{\hbar}[\mathbf{d} \cdot \mathbf{E}(\mathbf{r}, t)] \rho_{12}^{(2)}(\mathbf{r}, t, \theta, \varphi)+\frac{i}{\hbar}\left[\boldsymbol{\mu}_{12} \cdot \mathbf{E}(\mathbf{r}, t)\right]\left[\rho_{22}^{(2)}(\mathbf{r}, t, \theta, \varphi)-\rho_{11}^{(2)}(\mathbf{r}, t, \theta, \varphi)\right]
\end{aligned}
$$

As done previously, Eq. (38) is straightforwardly solved and $\rho_{12}^{(3)}(\mathbf{r}, t, \theta, \varphi)$ can be written as

$$
\begin{aligned}
& \rho_{12}^{(3)}(\mathbf{r}, t, \theta, \varphi)=\frac{i \mu_{12}}{8 \hbar^{3}} \sum_{\alpha, \beta, \gamma}\left\{\left\{\left(d^{2} a_{\alpha \beta}+2\left|\mu_{12}\right|^{2} d_{\alpha \beta}^{(1)}\right)\left[\epsilon_{\mu}(\theta, \varphi) \cdot \epsilon_{\alpha}\right]\left[\epsilon_{\mu}(\theta, \varphi) \cdot \epsilon_{\beta}\right]\left[\epsilon_{\mu}(\theta, \varphi) \cdot \epsilon_{\gamma}\right]+\frac{2}{3}\left|\mu_{12}\right|^{2} d_{\alpha \beta}^{(2)}\left(\epsilon_{\alpha} \cdot \epsilon_{\beta}\right)\left[\epsilon_{\mu}(\theta, \varphi) \cdot \epsilon_{\gamma}\right]\right\}\right. \\
& \times \frac{\mathscr{E}_{\alpha} \mathscr{E}_{\beta} \mathscr{E}_{\gamma}}{\left[\Gamma_{2}+i\left(\omega_{\alpha}+\omega_{\beta}+\omega_{\gamma}-\omega_{21}\right)\right]} \exp \left[i\left(\omega_{\alpha}+\omega_{\beta}+\omega_{\gamma}\right) t\right] \exp \left[-i\left(\mathbf{k}_{\alpha}+\mathbf{k}_{\beta}+\mathbf{k}_{\gamma}\right) \cdot \mathbf{r}\right] \\
& +\left\{\left(d^{2} b_{\alpha \beta}+d^{2} a_{\alpha \gamma}+2\left|\mu_{12}\right|^{2} e_{\alpha \beta}^{(1)}+2\left|\mu_{12}\right|^{2} d_{\alpha \gamma}^{(1)}\right)\left[\epsilon_{\mu}(\theta, \varphi) \cdot \epsilon_{\alpha}\right]\left[\epsilon_{\mu}(\theta, \varphi) \cdot \epsilon_{\beta}^{*}\right]\left[\epsilon_{\mu}(\theta, \varphi) \cdot \epsilon_{\gamma}\right]\right. \\
& \left.+\frac{2}{3}\left|\mu_{12}\right|^{2} e_{\alpha \beta}^{(2)}\left(\epsilon_{\alpha} \cdot \epsilon_{\beta}^{*}\right)\left[\epsilon_{\mu}(\theta, \varphi) \cdot \epsilon_{\gamma}\right]+\frac{2}{3}\left|\mu_{12}\right|^{2} d_{\alpha \gamma}^{(2)}\left(\epsilon_{\alpha} \cdot \epsilon_{\gamma}\right)\left[\epsilon_{\mu}(\theta, \varphi) \cdot \epsilon_{\beta}^{*}\right]\right\} \\
& \times \frac{\mathscr{E}_{\alpha} \mathscr{E}_{\beta}^{*} \mathscr{E}_{\gamma}}{\left[\Gamma_{2}+i\left(\omega_{\alpha}-\omega_{\beta}+\omega_{\gamma}-\omega_{21}\right)\right]} \exp \left[i\left(\omega_{\alpha}-\omega_{\beta}+\omega_{\gamma}\right) t\right] \exp \left[-i\left(\mathbf{k}_{\alpha}-\mathbf{k}_{\beta}+\mathbf{k}_{\gamma}\right) \cdot \mathbf{r}\right] \\
& +\left\{\left(d^{2} c_{\alpha \gamma}+d^{2} b_{\beta \alpha}+2\left|\mu_{12}\right|^{2} f_{\alpha \gamma}^{(1)}+2\left|\mu_{12}\right|^{2} e_{\beta \alpha}^{(1)}\right)\left[\epsilon_{\mu}(\theta, \varphi) \cdot \epsilon_{\alpha}^{*}\right]\left[\epsilon_{\mu}(\theta, \varphi) \cdot \epsilon_{\beta}\right]\left[\epsilon_{\mu}(\theta, \varphi) \cdot \epsilon_{\gamma}^{*}\right]\right. \\
& \left.+\frac{2}{3}\left|\mu_{12}\right|^{2} f_{\alpha \gamma}^{(2)}\left(\epsilon_{\alpha}^{*} \cdot \epsilon_{\gamma}^{*}\right)\left[\epsilon_{\mu}(\theta, \varphi) \cdot \epsilon_{\beta}\right]+\frac{2}{3}\left|\mu_{12}\right|^{2} e_{\beta \alpha}^{(2)}\left(\epsilon_{\alpha}^{*} \cdot \epsilon_{\beta}\right)\left[\epsilon_{\mu}(\theta, \varphi) \cdot \epsilon_{\gamma}^{*}\right]\right\} \\
& \times \frac{\mathscr{E}_{\alpha}^{*} \mathscr{E}_{\beta} \mathscr{E}_{\gamma}^{*}}{\left[\Gamma_{2}-i\left(\omega_{\alpha}-\omega_{\beta}+\omega_{\gamma}+\omega_{21}\right)\right]} \exp \left[-i\left(\omega_{\alpha}-\omega_{\beta}+\omega_{\gamma}\right) t\right] \exp \left[i\left(\mathbf{k}_{\alpha}-\mathbf{k}_{\beta}+\mathbf{k}_{\gamma}\right) \cdot \mathbf{r}\right] \\
& +\left\{\left(d^{2} c_{\alpha \beta}+2\left|\mu_{12}\right|^{2} f_{\alpha \beta}^{(1)}\right)\left[\epsilon_{\mu}(\theta, \varphi) \cdot \epsilon_{\alpha}^{*}\right]\left[\epsilon_{\mu}(\theta, \varphi) \cdot \epsilon_{\beta}^{*}\right]\left[\epsilon_{\mu}(\theta, \varphi) \cdot \epsilon_{\gamma}^{*}\right]\right. \\
& \left.+\frac{2}{3}\left|\mu_{12}\right|^{2} f_{\alpha \beta}^{(2)}\left(\epsilon_{\alpha}^{*} \cdot \epsilon_{\beta}^{*}\right)\left[\epsilon_{\mu}(\theta, \varphi) \cdot \epsilon_{\gamma}^{*}\right]\right\} \\
& \left.\times \frac{\mathscr{E}_{\alpha}^{*} \mathscr{E}_{\beta}^{*} \mathscr{E}_{\gamma}^{*}}{\left[\Gamma_{2}-i\left(\omega_{\alpha}+\omega_{\beta}+\omega_{\gamma}+\omega_{21}\right)\right]} \exp \left[-i\left(\omega_{\alpha}+\omega_{\beta}+\omega_{\gamma}\right) t\right] \exp \left[i\left(\mathbf{k}_{\alpha}+\mathbf{k}_{\beta}+\mathbf{k}_{\gamma}\right) \cdot \mathbf{r}\right]\right]
\end{aligned}
$$

From Eq. (37), $\rho_{22}^{(3)}(\mathbf{r}, t, \theta, \varphi)$ takes the form

$$
\begin{array}{r}
\rho_{22}^{(3)}(\mathbf{r}, t, \theta, \varphi)=\frac{i}{\hbar} \sum_{l, m} \int_{-\infty}^{t} d t_{1} \int_{0}^{2 \pi} d \varphi_{1} \int_{0}^{\pi} d \theta_{1} \sin \theta_{1}\left\{\rho_{12}^{(2)}\left(\mathbf{r}, t_{1}, \theta_{1}, \varphi_{1}\right)\left[\boldsymbol{\mu}_{21}\left(\theta_{1}, \varphi_{1}\right) \cdot \mathbf{E}\left(\mathbf{r}, t_{1}\right)\right]\right. \\
\left.-\left[\boldsymbol{\mu}_{12}\left(\theta_{1}, \varphi_{1}\right) \cdot \mathbf{E}\left(\mathbf{r}, t_{1}\right)\right] \rho_{21}^{2}\left(\mathbf{r}, t_{1}, \theta_{1}, \varphi_{1}\right)\right\} \\
\times Y_{l m}^{*}\left(\theta_{1}, \varphi_{1}\right) Y_{l m}(\theta, \varphi) \exp \left\{-\left[\Gamma_{1}+D l(l+1)\right]\left(t-t_{1}\right)\right\} .
\end{array}
$$

Introducing the expressions of $\rho_{12}^{(2)}(\mathbf{r}, t, \theta, \varphi)$ and $\rho_{21}^{(2)}(\mathbf{r}, t, \theta, \varphi)$ previously determined, $\rho_{22}^{(3)}(\mathbf{r}, t, \theta, \varphi)$ is given by the relation 


$$
\begin{aligned}
& \rho_{22}^{(3)}(\mathbf{r}, t, \theta, \varphi)=-\frac{i\left|\mu_{12}\right|^{2} d}{8 \hbar^{3}} \sum_{\alpha, \beta, \gamma}\left\{( a _ { \alpha \beta } - c _ { \alpha \beta } ^ { * } ) \left[\frac{G\left(\epsilon_{\alpha}, \epsilon_{\beta}, \epsilon_{\gamma}\right)}{5}\left[\frac{1}{\Gamma_{1}+2 D+i\left(\omega_{\alpha}+\omega_{\beta}+\omega_{\gamma}\right)}-\frac{1}{\Gamma_{1}+12 D+i\left(\omega_{\alpha}+\omega_{\beta}+\omega_{\gamma}\right)}\right)\right.\right. \\
& \left.+\frac{H\left(\boldsymbol{\epsilon}_{\alpha}, \boldsymbol{\epsilon}_{\beta}, \boldsymbol{\epsilon}_{\gamma}\right)}{\Gamma_{1}+12 D+i\left(\omega_{\alpha}+\omega_{\beta}+\omega_{\gamma}\right)}\right] \\
& \times \mathscr{E}_{\alpha} \mathscr{E}_{\beta} \mathscr{E}_{\gamma} \exp \left[i\left(\omega_{\alpha}+\omega_{\beta}+\omega_{\gamma}\right) t\right] \exp \left[-i\left(\mathbf{k}_{\alpha}+\mathbf{k}_{\beta}+\mathbf{k}_{\gamma}\right) \cdot \mathbf{r}\right]+\left(b_{\alpha \beta}+a_{\alpha \gamma}-c_{\alpha \gamma}^{*}-b_{\beta \alpha}^{*}\right) \\
& \times\left[\frac{G\left(\epsilon_{\alpha}, \epsilon_{\beta}^{*}, \epsilon_{\gamma}\right)}{5}\left[\frac{1}{\Gamma_{1}+2 D+i\left(\omega_{\alpha}-\omega_{\beta}+\omega_{\gamma}\right)}-\frac{1}{\Gamma_{1}+12 D+i\left(\omega_{\alpha}-\omega_{\beta}+\omega_{\gamma}\right)}\right]\right. \\
& \left.+\frac{H\left(\boldsymbol{\epsilon}_{\alpha}, \boldsymbol{\epsilon}_{\beta}^{*}, \boldsymbol{\epsilon}_{\gamma}\right)}{\Gamma_{1}+12 D+i\left(\omega_{\alpha}-\omega_{\beta}+\omega_{\gamma}\right)}\right] \\
& \times \mathscr{E}_{\alpha} \mathscr{E}_{\beta}^{*} \mathscr{E}_{\gamma} \exp \left[i\left(\omega_{\alpha}-\omega_{\beta}+\omega_{\gamma}\right) t\right] \exp \left[-i\left(\mathbf{k}_{\alpha}-\mathbf{k}_{\beta}+\mathbf{k}_{\gamma}\right) \cdot \mathbf{r}\right]+\left(c_{\alpha \gamma}+b_{\beta \alpha}-b_{\alpha \beta}^{*}-a_{\alpha \gamma}^{*}\right) \\
& \times\left[\frac{G\left(\epsilon_{\alpha}^{*}, \boldsymbol{\epsilon}_{\beta}, \boldsymbol{\epsilon}_{\gamma}^{*}\right)}{5}\left(\frac{1}{\Gamma_{1}+2 D-i\left(\omega_{\alpha}-\omega_{\beta}+\omega_{\gamma}\right)}-\frac{1}{\Gamma_{1}+12 D-i\left(\omega_{\alpha}-\omega_{\beta}+\omega_{\gamma}\right)}\right]\right. \\
& \left.+\frac{H\left(\boldsymbol{\epsilon}_{\alpha}^{*}, \boldsymbol{\epsilon}_{\beta}, \boldsymbol{\epsilon}_{\gamma}^{*}\right)}{\Gamma_{1}+12 D-i\left(\omega_{\alpha}-\omega_{\beta}+\omega_{\gamma}\right)}\right] \mathscr{E}_{\alpha}^{*} \mathscr{E}_{\beta} \mathscr{E}_{\gamma}^{*} \exp \left[-i\left(\omega_{\alpha}-\omega_{\beta}+\omega_{\gamma}\right) t\right] \exp \left[i\left(\mathbf{k}_{\alpha}-\mathbf{k}_{\beta}+\mathbf{k}_{\gamma}\right) \cdot \mathbf{r}\right] \\
& +\left(c_{\alpha \beta}-a_{\alpha \beta}^{*}\right)\left[\frac{G\left(\epsilon_{\alpha}^{*}, \epsilon_{\beta}^{*}, \epsilon_{\gamma}^{*}\right)}{5} \mid \frac{1}{\Gamma_{1}+2 D-i\left(\omega_{\alpha}+\omega_{\beta}+\omega_{\gamma}\right)}\right. \\
& \left.-\frac{1}{\Gamma_{1}+12 D-i\left(\omega_{\alpha}+\omega_{\beta}+\omega_{\gamma}\right)}\right) \\
& \left.+\frac{H\left(\epsilon_{\alpha}^{*}, \epsilon_{\beta}^{*}, \epsilon_{\gamma}^{*}\right)}{\Gamma_{1}+12 D-i\left(\omega_{\alpha}+\omega_{\beta}+\omega_{\gamma}\right)}\right] \\
& \left.\times \mathscr{E}_{\alpha}^{*} \mathscr{E}_{\beta}^{*} \mathscr{E}_{\gamma}^{*} \exp \left[-i\left(\omega_{\alpha}+\omega_{\beta}+\omega_{\gamma}\right) t\right] \exp \left[i\left(\mathbf{k}_{\alpha}+\mathbf{k}_{\beta}+\mathbf{k}_{\gamma}\right) \cdot \mathbf{r}\right]\right\},
\end{aligned}
$$

where $G\left(\epsilon_{\alpha}, \epsilon_{\beta}, \epsilon_{\gamma}\right)$ and $H\left(\epsilon_{\alpha}, \epsilon_{\beta}, \epsilon_{\gamma}\right)$ represent the quantities

$$
\begin{aligned}
\boldsymbol{G}\left(\boldsymbol{\epsilon}_{\alpha}, \boldsymbol{\epsilon}_{\beta}, \boldsymbol{\epsilon}_{\gamma}\right)= & \left(\boldsymbol{\epsilon}_{\beta} \cdot \boldsymbol{\epsilon}_{\gamma}\right)\left[\boldsymbol{\epsilon}_{\mu}(\theta, \varphi) \cdot \boldsymbol{\epsilon}_{\alpha}\right]+\left(\boldsymbol{\epsilon}_{\alpha} \cdot \boldsymbol{\epsilon}_{\gamma}\right)\left[\boldsymbol{\epsilon}_{\mu}(\theta, \varphi) \cdot \boldsymbol{\epsilon}_{\beta}\right] \\
& +\left(\boldsymbol{\epsilon}_{\alpha} \cdot \boldsymbol{\epsilon}_{\beta}\right)\left[\boldsymbol{\epsilon}_{\mu}(\theta, \varphi) \cdot \boldsymbol{\epsilon}_{\gamma}\right]
\end{aligned}
$$

and

$$
H\left(\boldsymbol{\epsilon}_{\alpha}, \boldsymbol{\epsilon}_{\beta}, \boldsymbol{\epsilon}_{\gamma}\right)=\left[\boldsymbol{\epsilon}_{\mu}(\theta, \varphi) \cdot \boldsymbol{\epsilon}_{\alpha}\right]\left[\boldsymbol{\epsilon}_{\mu}(\theta, \varphi) \cdot \boldsymbol{\epsilon}_{\beta}\right]\left[\boldsymbol{\epsilon}_{\mu}(\theta, \varphi) \cdot \boldsymbol{\epsilon}_{\gamma}\right]
$$

\section{MACROSCOPIC POLARIZATION}

We are now able to calculate the macroscopic polarization of the medium. At this end, we have to average over all possible orientations of the dipole moment. In the present work, we shall consider that the probability to find $\boldsymbol{\mu}(\theta, \varphi)$ in a solid angle $d \Omega=r^{2} d r d \varphi d \theta \sin \theta$ is a constant equal to $(1 / 4 \pi) d \Omega$. In other words, we consider a normalized uniform distribution of the dipole moment. This situation is generally encountered in many experiments concerning molecules in a solvent at room temperature. Let us note $\mathbf{P}^{(i)}(\mathbf{r}, t)$ the $i$ th-order averaged polarization term. $\mathbf{P}^{(i)}(\mathbf{r}, t)$ is expressed as

$$
\begin{aligned}
\mathbf{P}^{(i)}(\mathbf{r}, t)=\frac{1}{4 \pi} \int_{0}^{2 \pi} d \varphi \int_{0}^{\pi} d \theta \sin \theta[ & \mathbf{d}(\theta, \varphi) \rho_{22}^{(i)}(\mathbf{r}, t, \theta, \varphi) \\
& +\boldsymbol{\mu}_{12}(\theta, \varphi) \rho_{21}^{(i)}(\mathbf{r}, t, \theta, \varphi) \\
& \left.+\boldsymbol{\mu}_{21}(\theta, \varphi) \rho_{12}^{(i)}(\mathbf{r}, t, \theta, \varphi)\right]
\end{aligned}
$$

First, we calculate the first-order polarization. Introducing the expressions (18) and (20) in the expression (44), $\mathbf{P}^{(1)}(\mathbf{r}, t)$ takes the form

$$
\begin{aligned}
\mathbf{P}^{(1)}(\mathbf{r}, t)=\frac{i\left|\mu_{12}\right|^{2}}{6 \hbar} \sum_{\alpha} & {\left[\left(\frac{1}{\Gamma_{2}+i\left(\omega_{\alpha}+\omega_{21}\right)}-\frac{1}{\Gamma_{2}+i\left(\omega_{\alpha}-\omega_{21}\right)}\right] \epsilon_{\alpha} \mathscr{E}_{\alpha} \exp \left[i\left(\omega_{\alpha}-\mathbf{k}_{\alpha} \cdot \mathbf{r}\right)\right]\right.} \\
& \left.+\left[\frac{1}{\Gamma_{2}-i\left(\omega_{\alpha}-\omega_{21}\right)}-\frac{1}{\Gamma_{2}+i\left(\omega_{\alpha}+\omega_{21}\right)}\right] \epsilon_{\alpha}^{*} \mathscr{E}_{\alpha}^{*} \exp \left[-i\left(\omega_{\alpha}-\mathbf{k}_{\alpha} \cdot \mathbf{r}\right)\right]\right]
\end{aligned}
$$


Introducing the relations (24) and (28) in the expression (44), we are able to determine the second-order polarization $\mathbf{P}^{(2)}(\mathbf{r}, t)$. Then, $\mathbf{P}^{(2)}(\mathbf{r}, t)$ is zero, which is what could be expected from our model. This is due to the fact that we have considered a uniform distribution of the dipole moments. We are now ready to evaluate the third-order nonlinear polarization. This constitutes the main result of the present work. Starting with the relations (39) and (41) and introducing them into expression (44), $\mathbf{P}^{(3)}(\mathbf{r}, t)$ can be written as

$$
\begin{aligned}
\mathbf{P}^{(3)}(\mathbf{r}, t)=\sum_{\alpha, \beta, \gamma}\{ & \mathbf{A}_{\alpha \beta \gamma} \mathscr{E}_{\alpha} \mathscr{E}_{\beta} \mathscr{E}_{\gamma} \exp \left[i\left(\omega_{\alpha}+\omega_{\beta}+\omega_{\gamma}\right) t\right] \exp \left[-i\left(\mathbf{k}_{\alpha}+\mathbf{k}_{\beta}+\mathbf{k}_{\gamma}\right) \cdot \mathbf{r}\right] \\
& \left.\times \mathbf{B}_{\alpha \beta \gamma} \mathscr{E}_{\alpha} \mathscr{E}_{\beta}^{*} \mathscr{E}_{\gamma} \exp \left[i\left(\omega_{\alpha}-\omega_{\beta}+\omega_{\gamma}\right) t\right] \exp \left[-i\left(\mathbf{k}_{\alpha}-\mathbf{k}_{\beta}+\mathbf{k}_{\gamma}\right) \cdot \mathbf{r}\right]+\text { c.c. }\right\},
\end{aligned}
$$

where $\mathbf{A}_{\alpha \beta \gamma}$ and $\mathbf{B}_{\alpha \beta \gamma}$ are defined by the relations

$$
\begin{aligned}
\mathbf{A}_{\alpha \beta \gamma}= & \frac{i\left|\mu_{12}\right|^{2}}{8 \hbar^{3}}\left[\frac{d^{2} a_{\alpha \beta}+2\left|\mu_{12}\right|^{2} d_{\alpha \beta}^{(1)}-d^{2} c_{\alpha \beta}^{*}-2\left|\mu_{12}\right|^{2} f_{\alpha \beta}^{(1) *}}{\Gamma_{2}+i\left(\omega_{\alpha}+\omega_{\beta}+\omega_{\gamma}-\omega_{21}\right)}-d^{2} \frac{a_{\alpha \beta}-c_{\alpha \beta}^{*}}{\Gamma_{1}+2 D+i\left(\omega_{\alpha}+\omega_{\beta}+\omega_{\gamma}\right)}\right] \\
& \times \frac{\mathbf{A}\left(\epsilon_{\alpha}, \epsilon_{\beta}, \epsilon_{\gamma}\right)}{15}+\frac{2}{9} \frac{\left|\mu_{12}\right|^{2}\left(d_{\alpha \beta}^{(2)}-f_{\alpha \beta}^{(2) *}\right)\left(\epsilon_{\alpha} \cdot \epsilon_{\beta}\right) \epsilon_{\gamma}}{\Gamma_{2}+i\left(\omega_{\alpha}+\omega_{\beta}+\omega_{\gamma}-\omega_{21}\right)}
\end{aligned}
$$

and

$$
\begin{aligned}
\mathbf{B}_{\alpha \beta \gamma}=\frac{i\left|\mu_{12}\right|^{2}}{8 \hbar^{3}} \mid[ & \left(d^{2} b_{\alpha \beta}+d^{2} a_{\alpha \gamma}+2\left|\mu_{12}\right|^{2} e_{\alpha \beta}^{(1)}+2\left|\mu_{12}\right|^{2} d_{\alpha \gamma}^{(1)}\right) \frac{1}{15} \mathbf{A}\left(\boldsymbol{\epsilon}_{\alpha}, \boldsymbol{\epsilon}_{\beta}^{*}, \boldsymbol{\epsilon}_{\gamma}\right) \\
& \left.+\frac{2}{9}\left|\mu_{12}\right|^{2} e_{\alpha \beta}^{(2)}\left(\epsilon_{\alpha} \cdot \boldsymbol{\epsilon}_{\beta}^{*}\right) \boldsymbol{\epsilon}_{\gamma}+\frac{2}{9}\left|\mu_{12}\right|^{2} d_{\alpha \gamma}^{(2)}\left(\boldsymbol{\epsilon}_{\alpha} \cdot \boldsymbol{\epsilon}_{\gamma}\right) \epsilon_{\beta}^{*}\right] \frac{1}{\Gamma_{2}+i\left(\omega_{\alpha}-\omega_{\beta}+\omega_{\gamma}-\omega_{21}\right)} \\
& -\left[\left(d^{2} c_{\alpha \gamma}^{*}+d^{2} b_{\beta \alpha}^{*}+2\left|\mu_{12}\right|^{2} f_{\alpha \gamma}^{(1) *}+2\left|\mu_{12}\right|^{2} e_{\beta \alpha}^{(1) *}\right) \frac{1}{15} \mathbf{A}\left(\boldsymbol{\epsilon}_{\alpha}, \epsilon_{\beta}^{*}, \epsilon_{\gamma}\right)\right. \\
& \left.+\frac{2}{9}\left|\mu_{12}\right|^{2} f_{\alpha \gamma}^{(2) *}\left(\epsilon_{\alpha} \cdot \epsilon_{\gamma}\right) \epsilon_{\beta}^{*}+\frac{2}{9}\left|\mu_{12}\right|^{2} e_{\beta \alpha}^{(2) *}\left(\boldsymbol{\epsilon}_{\alpha} \cdot \epsilon_{\beta}^{*}\right) \epsilon_{\gamma}\right] \frac{1}{\Gamma_{2}+i\left(\omega_{\alpha}-\omega_{\beta}+\omega_{\gamma}+\omega_{21}\right)} \\
& \left.-d^{2}\left(b_{\alpha \beta}+a_{\alpha \gamma}-c_{\alpha \gamma}^{*}-b_{\beta \alpha}^{*}\right) \frac{1}{15} \mathbf{A}\left(\epsilon_{\alpha}, \epsilon_{\beta}^{*}, \epsilon_{\gamma}\right) \frac{1}{\Gamma_{1}+2 D+i\left(\omega_{\alpha}-\omega_{\beta}+\omega_{\gamma}\right)}\right]
\end{aligned}
$$

with

$$
\mathbf{A}\left(\boldsymbol{\epsilon}_{\alpha}, \boldsymbol{\epsilon}_{\beta}, \boldsymbol{\epsilon}_{\gamma}\right)=\left(\boldsymbol{\epsilon}_{\beta} \cdot \boldsymbol{\epsilon}_{\gamma}\right) \boldsymbol{\epsilon}_{\alpha}+\left(\boldsymbol{\epsilon}_{\alpha} \cdot \boldsymbol{\epsilon}_{\gamma}\right) \boldsymbol{\epsilon}_{\beta}+\left(\boldsymbol{\epsilon}_{\alpha} \cdot \boldsymbol{\epsilon}_{\beta}\right) \boldsymbol{\epsilon}_{\gamma} .
$$

Relation (46) combined with the definitions (47)-(49) gives a general expression of the third-order nonlinear polarization for an isotropic medium with permanent dipole moments and subject to rotational diffusion.

\section{DISCUSSION}

The expression of the nonlinear polarization previously obtained is general and can be applied to describe different nonlinear optical processes. Our purpose is to consider only degenerate four-wave mixing. Other processes will be discussed elsewhere. Therefore, we retain only the term $\mathbf{B}_{\alpha \beta \gamma}$, which gives the contributions to nonlinear processes in the direction $\mathbf{k}_{\alpha}-\mathbf{k}_{\beta}+\mathbf{k}_{\gamma}$. We assume that all fields have the same frequency. It must be noted that in our numerical simulations, we shall consider a situation corresponding to organic materials. This implies that the resonance frequency is chosen in the optical domain. The lifetime is assumed to be of the order of the nanosecond and we have taken a large pure dephasing rate as observed in these media. For the sake of simplicity, we have normalized all numerical results.

First we shall consider that all fields have parallel polarizations and that no rotational diffusion takes place in the medium (i.e., $D=0$ ). If we represent the imaginary part and the real part of $\mathbf{B}_{\alpha \beta \gamma}$ as a function of the field frequency $\omega$ for different values of $d\left(d=\mu_{22}-\mu_{11}\right)$, we first recover the well-known resonant degenerate fourwave-mixing process when $\omega$ is equal to the resonance frequency. This process is not very sensitive to the pres-

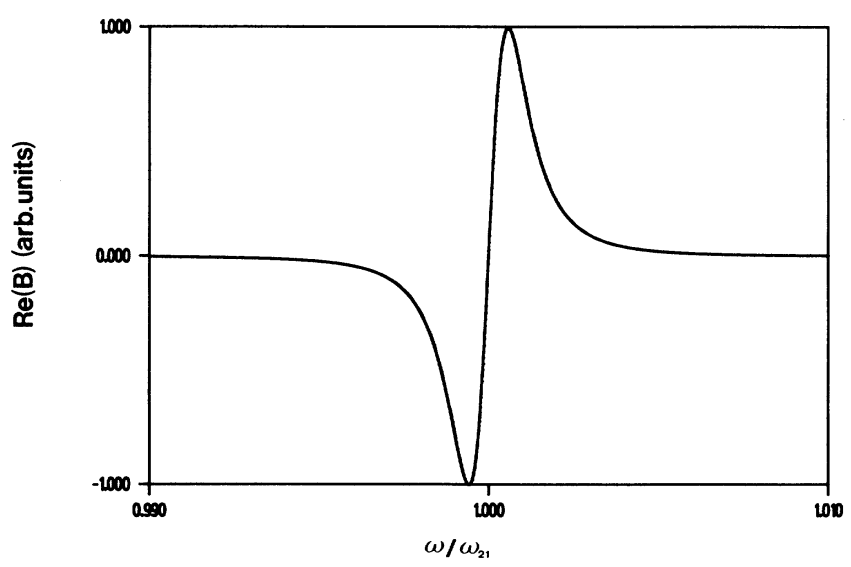

FIG. 1. Real part of the one-photon degenerate four-wavemixing nonlinear response. The values of the parameters are $\mu_{a b}=1, \Gamma_{2}=10^{3}, \Gamma_{1}=1, D=0$, and $\omega_{21}=10^{6}$. No variations are observed as $d$ varies. 


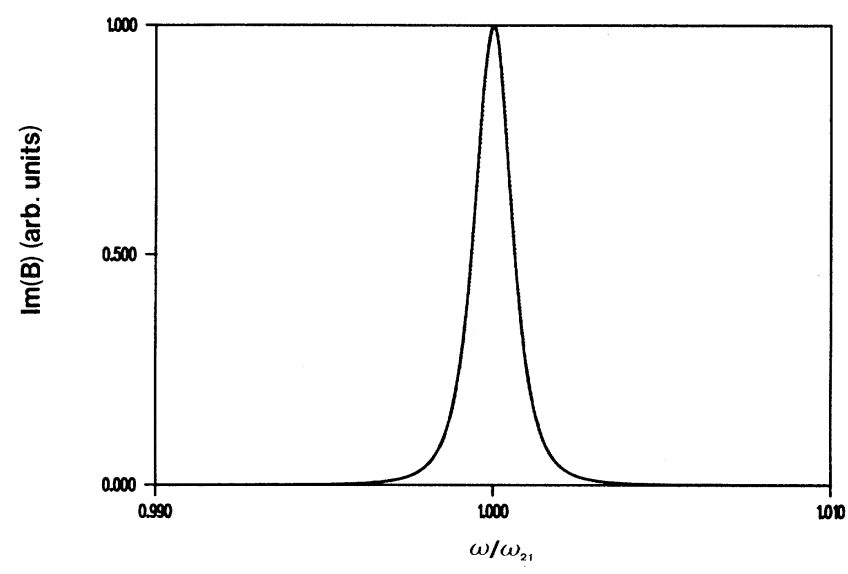

FIG. 2. Imaginary part of the one-photon degenerate fourwave-mixing nonlinear response. The value of the parameters are $\mu_{a b}=1, \Gamma_{2}=10^{3}, \Gamma_{1}=1, D=0$, and $\omega_{21}=10^{6}$. No variations are observed as $d$ varies.

ence of permanent dipole moments as shown in Figs. 1 and 2. On the other hand, we observe that a new process occurs when $\omega$ is equal to half of the resonance frequency. This process can be described as two-photon degenerate four-wave mixing and is dependent on the value of $d$. If $d$ is equal to zero, this process does not exist (Figs. 3 and 4). We observe that the imaginary part is negative. These points are consistent with results previously pub-

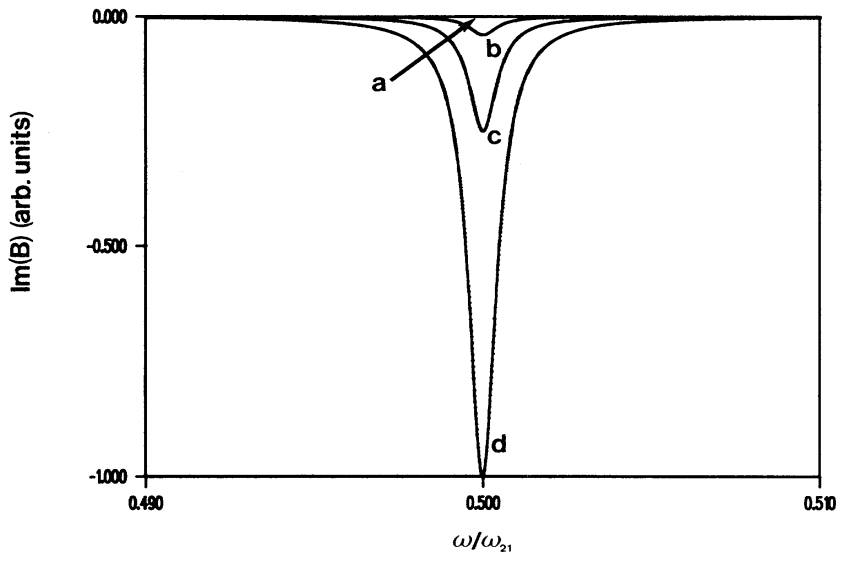

FIG. 4. Imaginary part of the two-photon degenerate fourwave-mixing nonlinear response. The values of the parameters are $\mu_{a b}=1, \Gamma_{2}=10^{3}, \Gamma_{1}=1, D=0, \omega_{21}=10^{6}$, and (a) $d=0$, (b) $d=2$, (c) $d=5$, and (d) $d=10$.

lished on two-photon phase conjugation $[7,8,13]$ or by comparison between such two-photon processes due to the presence of the permanent dipole moments and the Raman process $[14,15]$. It is interesting to analyze the term $\mathbf{B}_{\alpha \beta \gamma}$. It can be written into the form

$$
\mathbf{B}_{\alpha \beta \gamma}=\mathbf{C}_{\alpha \beta \gamma}+\mathbf{D}_{\alpha \beta \gamma},
$$

where

$$
\begin{aligned}
\mathbf{C}_{\alpha \beta \gamma}=\frac{i\left|\mu_{12}\right|^{2}}{8 \hbar^{3}} \mid[ & \left(d^{2} b_{\alpha \beta}+d^{2} a_{\alpha \gamma}+2\left|\mu_{12}\right|^{2} e_{\alpha \beta}^{(1)}+2\left|\mu_{12}\right|^{2} d_{\alpha \gamma}^{(1)}\right) \frac{1}{15} \mathbf{A}\left(\boldsymbol{\epsilon}_{\alpha}, \boldsymbol{\epsilon}_{\beta}, \boldsymbol{\epsilon}_{\gamma}\right) \\
& \left.+\frac{2}{9}\left|\mu_{12}\right|^{2} e_{\alpha \beta}^{(2)}\left(\boldsymbol{\epsilon}_{\alpha} \cdot \boldsymbol{\epsilon}_{\beta}^{*}\right) \boldsymbol{\epsilon}_{\gamma}+\frac{2}{9}\left|\mu_{12}\right|^{2} d_{\alpha \gamma}^{(2)}\left(\boldsymbol{\epsilon}_{\alpha} \cdot \boldsymbol{\epsilon}_{\gamma}\right) \boldsymbol{\epsilon}_{\beta}^{*}\right] \frac{1}{\Gamma_{2}+i\left(\omega_{\alpha}-\omega_{\beta}+\omega_{\gamma}-\omega_{21}\right)} \\
& -\left[\left(d^{2} c_{\alpha \gamma}^{*}+d^{2} b_{\beta \alpha}^{*}+2\left|\mu_{12}\right|^{2} f_{\alpha \gamma}^{(1) *}+2\left|\mu_{12}\right|^{2} e_{\beta \alpha}^{(1) *}\right) \frac{1}{15} \mathbf{A}\left(\boldsymbol{\epsilon}_{\alpha}, \boldsymbol{\epsilon}_{\beta}, \boldsymbol{\epsilon}_{\gamma}\right)\right. \\
& \left.\left.+\frac{2}{9}\left|\mu_{12}\right|^{2} f_{\alpha \gamma}^{(2) *}\left(\boldsymbol{\epsilon}_{\alpha} \cdot \boldsymbol{\epsilon}_{\gamma}\right) \boldsymbol{\epsilon}_{\beta}^{*}+\frac{2}{9}\left|\mu_{12}\right|^{2} e_{\beta \alpha}^{(2) *}\left(\boldsymbol{\epsilon}_{\alpha} \cdot \boldsymbol{\epsilon}_{\beta}^{*}\right) \boldsymbol{\epsilon}_{\gamma}\right] \frac{1}{\Gamma_{2}+i\left(\omega_{\alpha}-\omega_{\beta}+\omega_{\gamma}+\omega_{21}\right)}\right)
\end{aligned}
$$

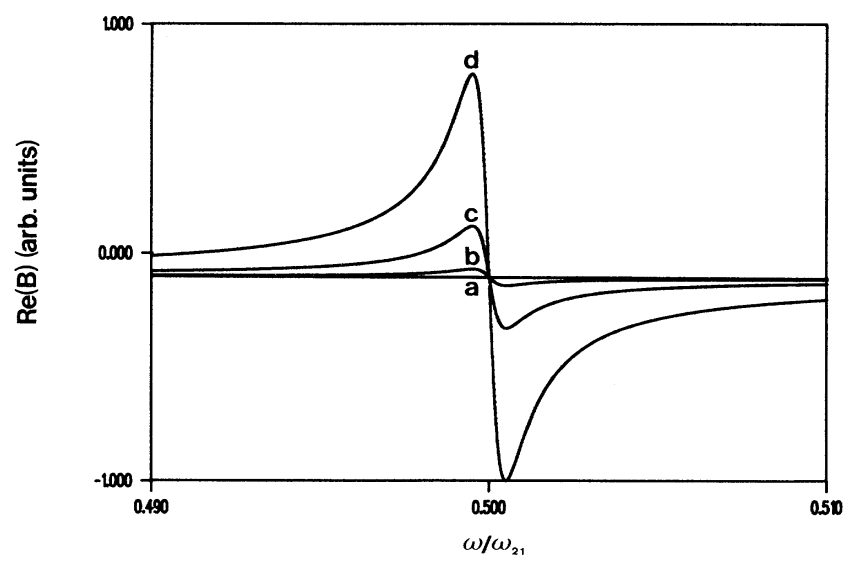

FIG. 3. Real part of the two-photon degenerate four-wavemixing nonlinear response. The values of the parameters are $\mu_{a b}=1, \Gamma_{2}=10^{3}, \Gamma_{1}=1, D=0, \omega_{21}=10^{6}$, and (a) $d=0$, (b) $d=2$, (c) $d=5$, and (d) $d=10$. and

$$
\begin{aligned}
\mathbf{D}_{\alpha \beta \gamma}=- & d^{2}\left(b_{\alpha \beta}+a_{\alpha \gamma}-c_{\alpha \gamma}^{*}-b_{\beta \alpha}^{*}\right) \\
& \times \frac{1}{15} \mathbf{A}\left(\boldsymbol{\epsilon}_{\alpha}, \boldsymbol{\epsilon}_{\beta}, \boldsymbol{\epsilon}_{\gamma}\right) \frac{1}{\Gamma_{1}+2 D+i\left(\omega_{\alpha}-\omega_{\beta}+\omega_{\gamma}\right)} .
\end{aligned}
$$

$\mathbf{C}_{\alpha \beta \gamma}$ is due to the third-order coherence of the medium while $\mathbf{D}_{\alpha \beta \gamma}$ is due to the third-order population. This last quantity results from second-order coherences which are generated by a two-photon process. $\mathbf{D}_{\alpha \beta \gamma}$ can be schematized by the perturbation chain

$$
\rho_{11}^{(0)} \rightarrow\left[\begin{array}{l}
\rho_{21}^{(1)} \\
\rho_{12}^{(1)}
\end{array}\right] \rightarrow\left(\begin{array}{l}
\rho_{21}^{(2)} \\
\rho_{12}^{(2)}
\end{array}\right) \rightarrow\left(\begin{array}{l}
\rho_{22}^{(3)} \\
\rho_{11}^{(3)}
\end{array}\right)
$$

$\mathbf{C}_{\alpha \beta \gamma}$ is different and can be represented by the association of two perturbation chains, 
(1) $\rho_{11}^{(0)} \rightarrow\left[\begin{array}{l}\rho_{21}^{(1)} \\ \rho_{12}^{(1)}\end{array}\right] \rightarrow\left[\begin{array}{l}\rho_{21}^{(2)} \\ \rho_{12}^{(2)}\end{array}\right] \rightarrow\left[\begin{array}{l}\rho_{21}^{(3)} \\ \rho_{12}^{(3)}\end{array}\right)$,

(2) $\rho_{11}^{(0)} \rightarrow\left[\begin{array}{c}\rho_{21}^{(1)} \\ \rho_{12}^{(1)}\end{array}\right] \rightarrow\left[\begin{array}{l}\rho_{22}^{(2)} \\ \rho_{11}^{(2)}\end{array}\right] \rightarrow\left[\begin{array}{l}\rho_{21}^{(3)} \\ \rho_{12}^{(3)}\end{array}\right]$.

The first chain does not include the creation of population. It is a pure coherent two-photon process. The second chain includes the creation of population. We have numerically tested the contributions of each chain. This can be easily done by taking $e_{\alpha \beta}^{(1)}, e_{\alpha \beta}^{(2)}, d_{\alpha \beta}^{(1)}, d_{\alpha \beta}^{(2)}, f_{\alpha \beta}^{(1)}$, and $f_{\alpha \beta}^{(2)}$ equal to zero in the expression of $\mathbf{C}_{\alpha \beta \gamma}$. In this case, we consider only the coherent two-photon process. We observed that numerical results are not modified. In other words, Figs. 3 and 4 are identical in this case to the one where we consider all processes. This shows clearly that the contributions of the second chain previously described do not contribute significantly to the general process. Therefore, we can neglect all processes involving the creation of second-order population. We recover here a situation similar to the one observed in twophoton degenerate four-wave mixing in three-level systems.

Figures 5 and 6 show the influence of the rotational diffusion. We see that the effects of orientational averaging are completely different from those observed in the usual one-photon degenerate four-wave mixing, as the one obtained when the field frequencies are equal to the resonance frequency. This situation is illustrated in Figs. 7 and 8. In this case, the consequence of orientational averaging is to weaken the nonlinear effect. This results from the fact that third-order density-matrix terms are generated by second-order populations that depend on the rotational diffusion. If we consider the two-photon degenerate four-wave mixing, the third-order densitymatrix terms are coming from second-order coherences that do not depend on the rotational diffusion because in organic materials the dephasing rate $\Gamma_{2}$ is much greater

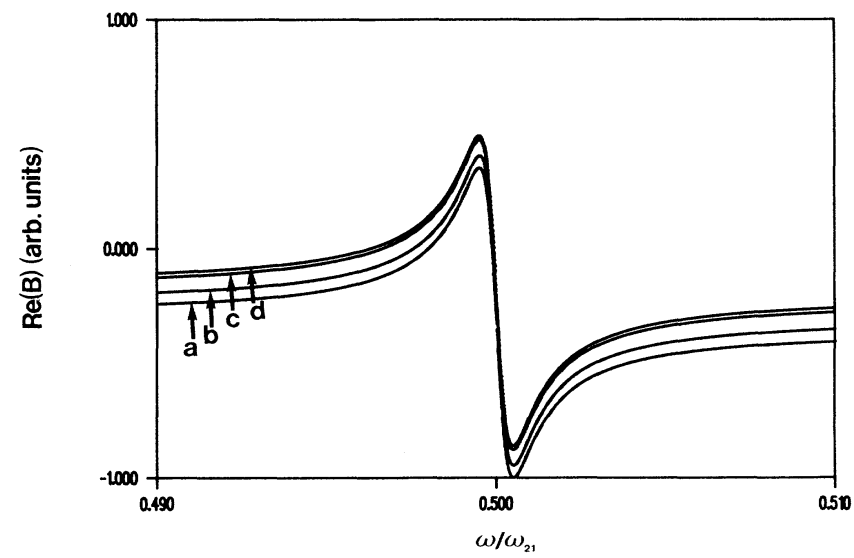

FIG. 5. Influence of the rotational diffusion on the real part of the two-photon degenerate four-wave-mixing nonlinear response. The values of the parameters are $\mu_{a b}=1, \Gamma_{2}=10^{3}$, $\Gamma_{1}=1, d=5, \omega_{21}=10^{6}$, and (a) $D=0$, (b) $D=0.1$, (c) $D=1$, and (d) $D=10$.

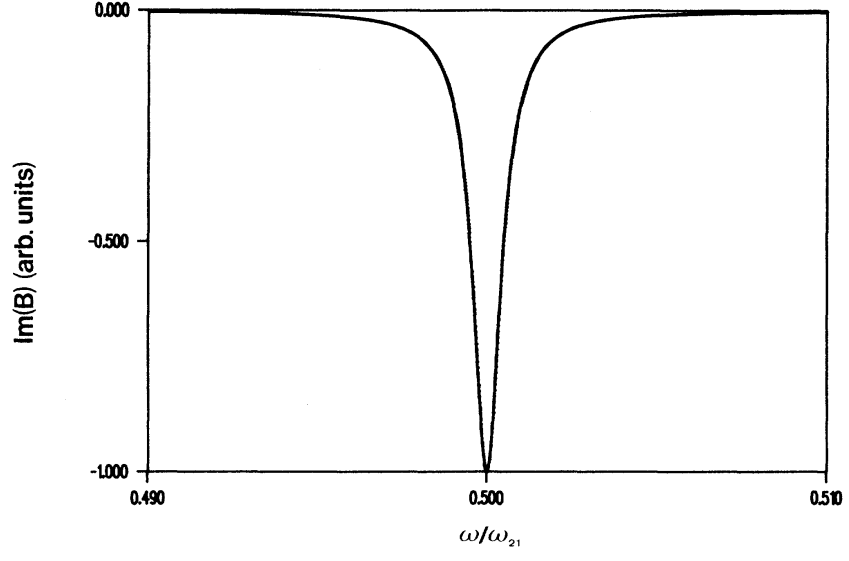

FIG. 6. Influence of the rotational diffusion on the imaginary part of the two-photon degenerate four-wave-mixing nonlinear response. The values of the parameters are $\mu_{a b}=1, \Gamma_{2}=10^{3}$, $\Gamma_{1}=1, d=5$, and $\omega_{21}=10^{6}$. No dependence with respect to $D$ is observed.

than $D$. Because of this realistic assumption rotational diffusion will have no effect in the case of the two-photon degenerate four-wave-mixing process. This situation is observed in Figs. 5 and 6 . The small variations corresponding to the $D$ dependence and observed on the real part result from contributions due to the real part of the one-photon degenerate four-wave mixing, which still persist in this spectral range in the case of our numerical simulation. These variations do not appear on the imaginary part because the imaginary part of the one-photon degenerate four-wave mixing decreases more rapidly than the real part and its influence is less important. This is confirmed by the fact that if we take the quantities $e_{\alpha \beta}^{(1)}$, $e_{\alpha \beta}^{(2)}, d_{\alpha \beta}^{(1)}, d_{\alpha \beta}^{(2)}, f_{\alpha \beta}^{(1)}$, and $f_{\alpha \beta}^{(2)}$ equal to zero, we no longer observe a $D$ dependence in the two-photon degenerate four-wave-mixing process.

Finally, Figs. 9 and 10 show the nonlinear response for different types of field polarizations. We have considered two different situations where either all fields have parallel polarizations or the fields having the wave vectors $\mathbf{k}_{\alpha}$ and $\mathbf{k}_{\gamma}$ have parallel polarizations but are cross polarized with respect to the field having the wave vector $\mathbf{k}_{\beta}$. This last situation is generally encountered in phaseconjugation experiments or in forward degenerate fourwave-mixing experiments when the pump fields are cross polarized with respect to the probe field. The evolution of the nonlinear response between these two situations is quite similar to the one observed in the well-known resonant degenerate four-wave mixing. The vertical translation observed in Fig. 10 as a function of $D$ still results from the contribution of the one-photon degenerate four-wave-mixing process.

Bavli and Band [16] have calculated the third-order nonlinear absorption and dispersion of a homogeneously broadened two-level system with permanent dipole moments. They analyzed the nonlinear susceptibilities $\chi^{(3)}(-\omega ; \omega,-\omega, \omega)$ and $\chi^{(3)}\left(-\omega_{1} ; \omega_{2},-\omega_{2}, \omega_{1}\right)$. They show that the $\chi^{(3)}(-\omega ; \omega,-\omega, \omega)$ spectrum has two features, one at frequency $\omega_{b a}$ dominated by contribu- 


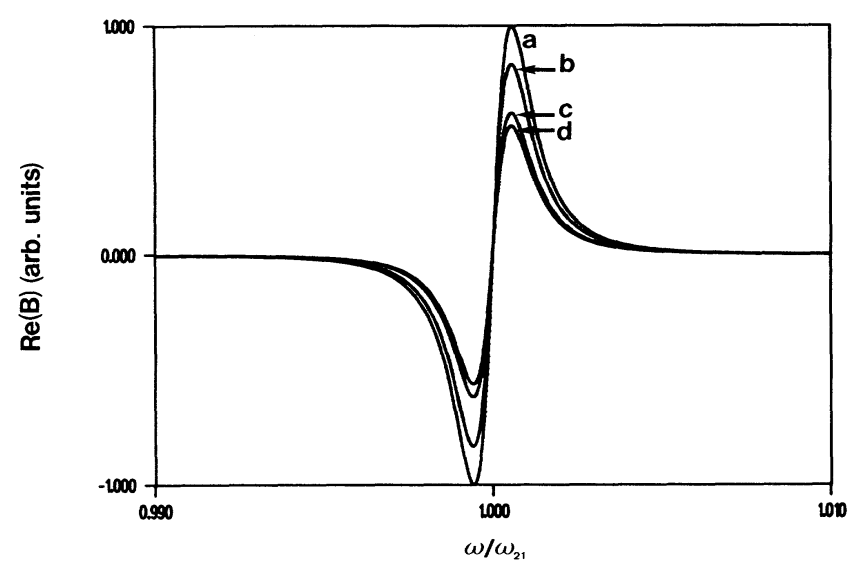

FIG. 7. Influence of the rotational diffusion on the real part of the one-photon degenerate four-wave-mixing nonlinear response. The values of the parameters are $\mu_{a b}=1, \Gamma_{2}=10^{3}$, $\Gamma_{1}=1, d=5, \omega_{21}=10^{6}$, and (a) $D=0$, (b) $D=0.1$, (c) $D=1$, and (d) $D=10$.

tions proportional to $\mu_{b a}^{4}$, where $\mu_{b a}$ is the transition dipole moment of the two levels with transition frequency $\omega_{b a}$, and the other at frequency $\omega_{b a} / 2$ dominated by contributions proportional to $(\Delta \mu)^{2} \mu_{b a}^{2}$ where $\Delta \mu$ is the difference between the permanent dipole moments of the levels. They also demonstrate that sharp features in $\chi^{(3)}\left(-\omega_{1} ; \omega_{2},-\omega_{2}, \omega_{1}\right)$ appear when $\omega_{2}$ is near $\omega_{b a}, \omega_{1}$, $\omega_{1}+\omega_{b a}$, and $\left|\omega_{1}-\omega_{b a}\right|$. These results are consistent with ours, but their theoretical model does not take into account orientational diffusion processes and field polarizations that have been introduced here.

\section{CONCLUSION}

We have presented a general expression of the thirdorder nonlinear polarization for an isotropic medium

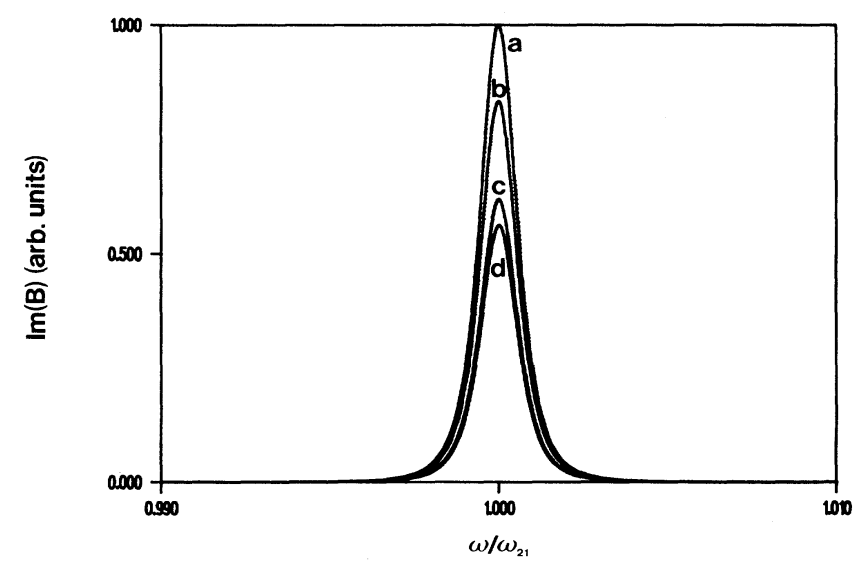

FIG. 8. Influence of the rotational diffusion on the imaginary part of the one-photon degenerate four-wave-mixing nonlinear response. The values of the parameters are $\mu_{a b}=1, \Gamma_{2}=10^{3}$, $\Gamma_{1}=1, d=5, \omega_{21}=10^{6}$, and (a) $D=0$, (b) $D=0.1$, (c) $D=1$, and (d) $D=10$.

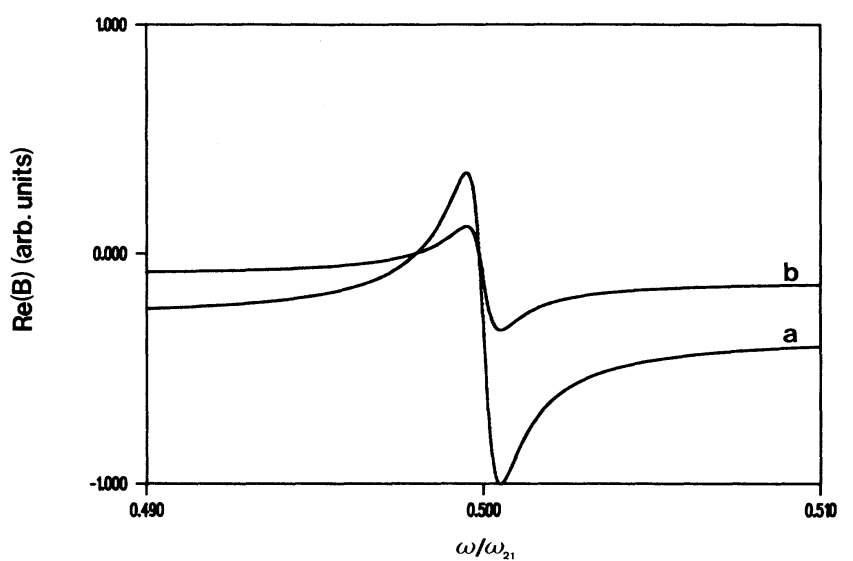

FIG. 9. Influence of the field polarization on the real part of the two-photon degenerate four-wave-mixing nonlinear response. The values of the parameters are $\mu_{a b}=1, \Gamma_{2}=10^{3}$, $\Gamma_{1}=1, d=5, \omega_{21}=10^{6}$, and $D=0$ : (a) parallel polarizations; (b) perpendicular polarizations.

modeled by homogeneously broadened two-level systems having unequal permanent dipole moments and subject to rotational diffusion. This theoretical model has been used to study a two-photon degenerate four-wave-mixing process that is a consequence of the existence of the permanent dipole moments. The influence of these permanent dipole moments, as well as the ones of the rotational diffusion and field polarizations, have been analyzed. This nonlinear process seems interesting for two reasons. First, it must be possible to determine the value of $d$ by a four-wave-mixing experiment since the twophoton degenerate four-wave-mixing process does not exist if the permanent dipole moments are equal or zero. If we consider a phase-conjugation experiment with fields having frequencies equal to half of the resonance frequency of the medium, the intensity of the conjugated wave

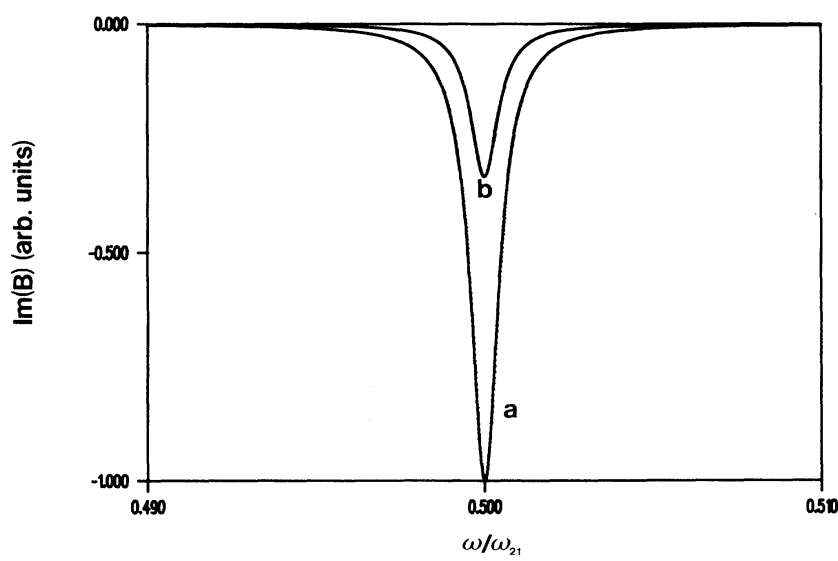

FIG. 10. Influence of the field polarization on the imaginary part of the two-photon degenerate four-wave-mixing nonlinear response. The values of the parameters are $\mu_{a b}=1, \Gamma_{2}=10^{3}$, $\Gamma_{1}=1, d=5, \omega_{21}=10^{6}$, and $D=0$ : (a) parallel polarizations; (b) perpendicular polarizations. 
will be depending on the magnitude of $d$. The second point of interest is the fact that this process occurs in a spectral domain where the medium is transparent. This can be very useful in phase-conjugation experiments where it will be possible to take different thicknesses of media. On the other hand, no thermal grating will appear in this process. The theoretical treatment which has been developed in this paper can be used to study other nonlinear processes. The analytical expressions show clearly that the permanent dipole moment also has an influence on the generation of the third harmonic and in nondegenerate four-wave-mixing processes. These effects are planned to be discussed elsewhere.

\section{ACKNOWLEDGMENTS}

The Institut de Physique et Chimie des Matériaux de Strasbourg is "unité mixte No. 380046 du Centre National de la Recherche Scientifique, de l'Université Louis Pasteur et de l'Ecole Européenne des Hautes Etudes des Industries Chimiques de Strasbourg." We wish to thank Dr. A. Boeglin for helpful comments and discussions.
[1] M. A. Kmetic and W. J. Meath, Phys. Lett. A 108, 340 (1985).

[2] W. J Meath and E. A. Power, J. Phys. B 17, 763 (1984).

[3] M. A. Kmetic and W. J. Meath, Phys. Rev. A 41, 1556 (1990).

[4] M. A. Kmetic, R. A. Thuraisingham, and W. J. Meath, Phys. Rev. A 33, 1688 (1986).

[5] G. F. Thomas, Phys. Rev. A 33, 1033 (1986).

[6] Y. B. Band, R. Bavli, and D. F. Heller, Chem. Phys. Lett. 156, 405 (1989).

[7] R. Bavli, D. F. Heller, and Y. B. Band, Phys. Rev. A 41, 3960 (1990).

[8] T. Y. Fu and M. Sargent III, Opt. Lett. 5, 433 (1980).

[9] M. Kauranen, D. J. Gauthier, M. S. Malcuit, and R. W.
Boyd, Phys. Rev. A 40, 1908 (1989).

[10] B. Dick and G. Hohlneicher, J. Chem. Phys. 76, 5755 (1982).

[11] B. S. Wherett, A. L. Smirl, and T. F. Boggess, IEEE J. Quantum Electron. QE-19, 680 (1983).

[12] J. P. Lavoine and A. A. Villaeys, Phys. Rev. A 39, 1922 (1989).

[13] J. P. Lavoine, A. Boeglin, S. H. Lin, and A. A. Villaeys, Phys. Rev. A 38, 2896 (1988).

[14] Optical Phase Conjugation, edited by R. A. Fisher (Academic, New York, 1982).

[15] B. E. Scharf and Y. B. Band, Chem. Phys. Lett. 144, 165 (1988).

[16] R. Bavli and Y. B. Band, Phys. Rev A 43, 5039 (1991). 